\title{
Improved agarose gel assay for quantification of growth factor-induced cell motility
}

\author{
Yoanne Mousseau, Dany Leclers, Karine Faucher-Durand, Jeanne Cook-Moreau, \\ Anne-Sophie Lia-Baldini, Michel Rigaud, and Franck G. Sturtz
}

BioTechniques 43:509-516 (October 2007)

doi $10.2144 / 000112557$

\begin{abstract}
Cell chemotaxis is frequently required in normal or pathological situations such as invasion, metastasis, and tumor angiogenesis and may involve many different cell types. At present, no device can simultaneously (i) make morphological observations, (ii) quantify cell migration, (iii) test multiple chemoattracting gradients, and (iv) analyze cell-cell interactions. We developed an agarose-based assay to address these questions. Two glass molds were designed, around which agarose gel could be poured to form specific well shapes. Using a vital nuclear stain (Hoechst 33258), we characterized the migration profile of adherent or suspension cells. Cells could be observed during the entire migration process. We were able to follow cells moving toward chemoattractants or being repulsed by other molecules, and we could estimate average migration speed. Using this inexpensive assay, we were able to obtain precise, reproducible results concerning the chemotactic behavior of different cell types. The resulting data differentiated between chemokinetic and chemotactic movement. Chemotactic potencies could be compared using different criteria, such as the number of attracted cells, induced speed, and morphological aspect. This improved agarose assay appears to be a reliable and inexpensive alternative to other available chemotaxis study tools.
\end{abstract}

\section{Introduction}

Mural cell motility, for cells such as pericytes and vascular smooth muscle cells (VSMCs) is frequently required for pathological processes such as tumor angiogenesis or atherosclerosis (1-4). In order to relate experimental in vitro results to in vivo conditions, it is crucial to use the appropriate experimental approach to elucidate the mechanisms driving cell migration. Among the variety of systems used to assess chemotaxis, the most commonly used is the modified Boyden chamber assay and its variations (5-8). It consists of two superposed cell culture chambers separated by a filter that allows cells to pass through. Once the cells placed in the upper chamber have migrated through pores in the filter to the lower one, they are collected and counted. It is a prevailing method to assess the number of cells attracted by chemoattractant (9), and it is easy to differentiate chemokinetic and chemotactic movements (10). The Zigmond chamber is a glass slide in which two wells are separated by a bridge where cells are seeded (11). It was designed for direct viewing of small amounts of cells (12). Similar to the Zigmond chamber, the Dunn chamber was developed to address chemoattractant gradient prediction and experimental reliability for slowly migrating cells $(13,14)$. Since the late 1970 s, different under-agarose assays have been developed to assess chemotaxis (15). With this technique, cells are placed in wells cut in the agarose gel or on the surface of the gel $(16,17)$. The underagarose assay has mainly been used for studying blood cells such as neutrophils (18-20). Chemotaxis studies of other cell types using the agarose assay are rare $(21,22)$ mainly because of the difficulty of data analysis due to the agarose gel, which makes cell observation difficult.

In our case, we needed a tool that enabled us to understand and to precisely describe VSMC chemotactic behavior. For this reason, our experimental approach had to fulfill the following expectations. We wanted to be able to (i) observe the cells at any time; (ii) quantify induced migration; (iii) compare the effect of different factors; and (iv) analyze cellular interactions. Although the modified Boyden chamber is still the most popular chemotaxis study tool, as it is available in multi-well plates, giving ready-to-use and reproducible experimental conditions, it is difficult to follow cells during their migration (23) and to evaluate the influence of gravity since it employs vertical displacement. Moreover, cells cannot be observed microscopically or immunostained during a particular step of the migration. In the case of the Zigmond or Dunn chambers, one chamber is needed for each experimental condition, which makes this a long and expensive procedure when many conditions are tested.

Agarose gels are simple to make, can be modified to specific experimental needs, and are quite inexpensive compared to other commercial devices. Therefore, we developed a strategy that uses glass molds to create particular agarose patterns that allow for the 


\section{Research Reports}

study of adherent or nonadherent cell migration and cell attraction by another cell type. With this assay, we observed the behavior of different cell types toward growth factors, such as Plateletderived growth factor (PDGF-BB) and basic fibroblast growth factor (bFGF), or toward secreted factors contained in the supernatant of bovine capillary endothelial cells (EJG) and tumor cells (1547 cells). We show that this assay permits differentiation between chemokinetic and chemotactic movements of different cell types and comparison of the chemotactic potencies of growth factors using criteria such as the number of attracted cells and migration speed.

\section{MATERIALS AND METHODS}

\section{Cell Culture and Chemicals}

PDGF-BB was purchased from R\&D Systems (Lille, France). Sphingosine-1-phosphate and FGF2 were purchased from Sigma-Aldrich (Lyon, France). Human erythroleukemia cells (HEL), osteosarcoma cells (1547), and bovine endothelial cells (EJG) were obtained from ATCC (Manassas, VA, USA). T lymphocytes were isolated from total human blood by Ficoll ${ }^{\circledR}$ gradient density centrifugation (histopaque 1077; SigmaAldrich) and the Pan T cell isolation kit (Miltenyi Biotec, Bergisch Gladbach, Germany). VSMCs were obtained by explants of thoracic aorta from 3-weekold male Sprague-Dawley rats using a method adapted from Sachinidis et al. (24). After four passages, cells were immunostained with an anti- $\alpha$-smooth muscle actin antibody (anti- $\alpha$ SMA; Sigma-Aldrich) to characterize the cells as smooth muscle cells. Rat fibroblasts were isolated from explants of thoracic lymphoid duct and labeled with an anti-fibroblast surface protein antibody (Sigma-Aldrich). Primary cultured cells, VSMCs, and fibroblasts were used for our experiments between passages 5-15. All cells were cultured in Dulbecco's modified Eagle's medium (DMEM) supplemented with 10\% heat-inactivated fetal bovine serum (FBS; BioWhittaker, Walkersville, MD, USA). These cultures were maintained
A

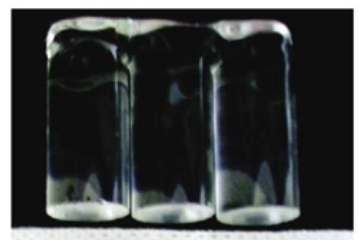

B
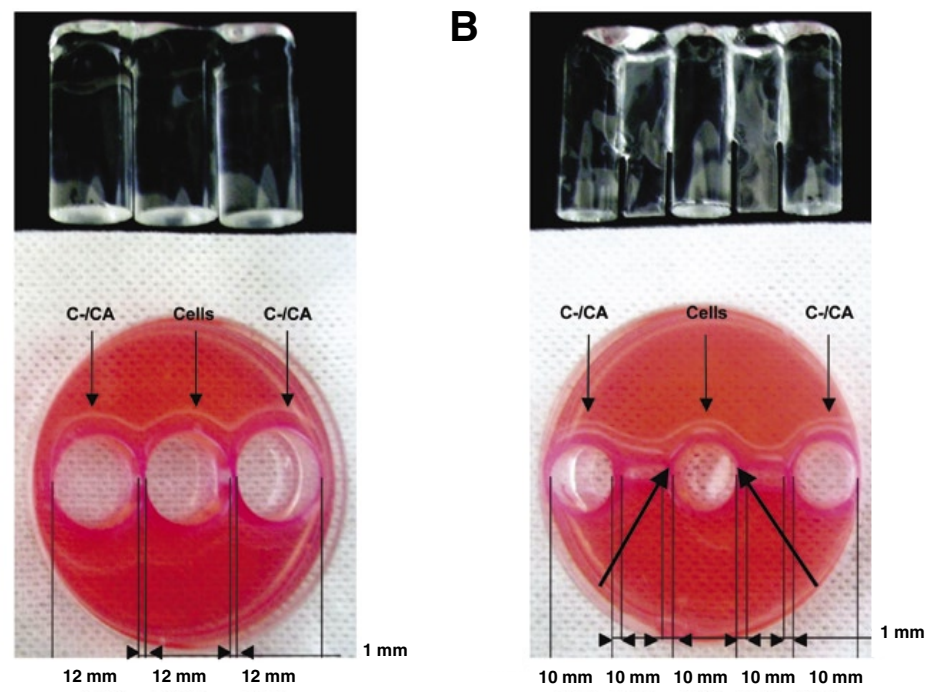

Figure 1. Glass molds used to produce wells in the agarose gel. Cells were seeded in the central well and migrated toward the two lateral wells if they were attracted by chemoattractant (CA) or negative control (FBS-free DMEM; C-) (A) In this configuration, the wells are only separated by $1 \mathrm{~mm}$ agarose gel. (B) In the more complex shape, the wells are separated by a $1.5-\mathrm{mm}$ and $10-\mathrm{mm}$-long wide corridor closed by $1-\mathrm{mm}$-thick agarose wall at each end. The black arrows indicate the agarose membranes which are cut with a scalpel $24 \mathrm{~h}$ after the cells are seeded to enable chemoattractant diffusion and cell migration in the corridors. FBS, fetal bovine serum; DMEM, Dulbecco's modified Eagle's medium.

at $37^{\circ} \mathrm{C}$ in $5 \% \mathrm{CO}_{2}$, passaged prior to confluence, and fed twice weekly.

\section{Agarose Gel Well Formation}

We designed two glass molds intended to be placed in a Petri dish that is subsequently filled with agarose. When removed, these molds form three wells separated by a thin barrier of agarose (Figure 1). The glass molds were made by a glass blower and can be sterilized by autoclaving for repeated use. A $1 \%$ solution of agarose was prepared in medium composed of $50 \%$ phosphate-buffered saline (PBS) and 50\% DMEM (Gibco ${ }^{\circledR}$; Invitrogen, Carlsbad, CA, USA) supplemented with $10 \%$ heat-inactivated FBS and 2 $\mathrm{mM}$ L-glutamine (Invitrogen). For a 60-mm diameter Petri dish, $10 \mathrm{~mL}$ final agarose solution were needed. One hundred micrograms of type II agarose (Sigma-Aldrich) were added to 50 $\mathrm{mL}$ PBS. After agarose was dissolved in PBS in a microwave oven, $50 \mathrm{~mL}$ DMEM were added. The agarose solution was poured into the Petri dish around the specific glass piece used to give the well shape (Figure 1). After 20-30 min of cooling, the gel was humidified with $5 \mathrm{~mL}$ DMEM, and the glass template was removed. Before performing the cell assay, $5 \mathrm{~mL}$ FBSfree DMEM were added to the gel for $1-6 \mathrm{~h}$ in order to stabilize the $\mathrm{pH}$, for saturation of the gel and to prevent culture medium from diffusing in the gel during the experiment.

\section{Chemotaxis Assay and Measurements During Cell Migration}

Cells were seeded at a density of $80 \times 10^{3}$ cells per well for adherent cells (Figure 2A), and $100 \times 10^{3}$ cells per well for cells in suspension (Figure 2B) with 10\% FBS-DMEM. After $24 \mathrm{~h}$, the medium was removed and replaced by FBS-free DMEM or 1\% FBS-DMEM depending on the cell type. Molecules tested for their chemoattractant activity were then placed in one of the neighboring wells, with FBS-free DMEM placed in the other well as a negative control. Alternatively, the effects of two different molecules could be compared by placing one in each well. Image capture and measurements were accomplished using a Leica DM IRB microscope linked to NIS-Elements imaging software (Nikon Instruments, Melville, NY, USA) via a charged- 


\section{Research Reports}

A
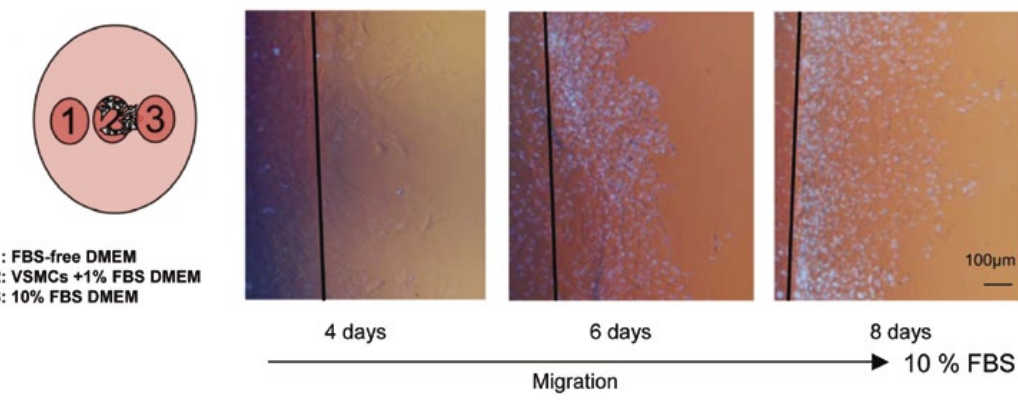

B
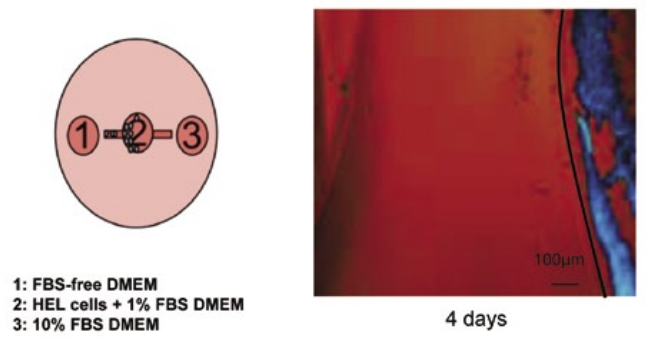

4 days

Negative control

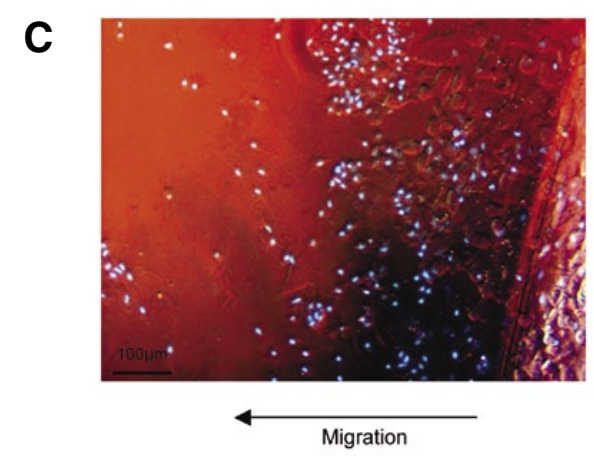

D

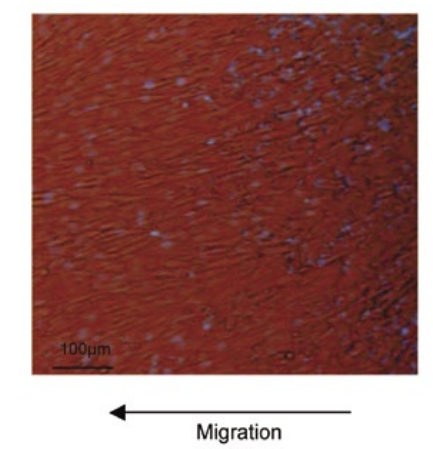

Figure 2. Choice of the adapted well shape and cell behavior. Cells could be observed during migration even through the agarose gel due to nuclear labeling with Hoechst 33258. The interface between agarose wall and medium is shown by a black line. (A) Vascular smooth muscle cells (VSMCs) migrated toward the $10 \%$ fetal bovine serum (FBS) source after 4-8 days of stimulation. (B) After 4-6 days, human erythroleukemia cells (HEL) migrated along the corridor separating the wells in the opposite direction from the FBS gradient and accumulated at the border of the negative control well. (C) VSMCs adopt a compact and circular morphology as they transverse the agarose barrier. (D) VSMCs polarization toward chemoattracting gradient.

coupled device (CCD) camera. Cell nuclei were labeled according to $\mathrm{Li}$ et al. (25) using $50 \mathrm{ng} / \mathrm{mL}$ Hoechst 33258 (Sigma-Aldrich), which was added 30 min prior to cell observations.

The number of migrating cells $(N)$ represented the difference between cells moving toward chemoattractant [chemotaxis (CT)], counted after they reached the reference line (Figure 3A), versus cells moving toward the negative control [chemokinesis (CK)].

$$
N=\mathrm{CT}-\mathrm{CK}
$$

When more than 100 cells were present in CT or CK zones, cells were counted in three randomly chosen fields of $100 \mu \mathrm{m}^{2}$ using NIS software. The average number in $100 \mu \mathrm{m}^{2}$ was then used to calculate the total cell number in the whole area occupied by migrating cells.
To estimate cell speed, the border of the well where the cells were seeded was considered as the reference line. Global estimated cell speed $(S)$ was calculated considering the median distance between the front of migrating cells $\left(D_{m}\right)$ (Figure $4 \mathrm{~A}$ ), divided by the duration $(t)$ of the experiment.

$$
\left.S(\mu \mathrm{m} / \text { days })=D_{m}(\mu \mathrm{m}) / t \text { (days }\right)
$$

[Eq. 2]

For each measured parameter, the value corresponds to the mean of three independent experiments.

\section{Statistical Analysis}

Statistical analyses were performed using StatView ${ }^{\circledR} 5.0$ software (SAS Institute, Cary, NC, USA). Mean comparisons were first done using the nonparametric Kruskal-Wallis (KW) test for three or more parameters. When the KW test indicated a significant difference (i.e., $P<0.05$ ), the nonparametric Mann-Withney test was further performed for the mean comparison of two parameters.

\section{RESULTS}

In order to obtain standard experimental conditions, we developed an agarose-based migration assay by pouring an agarose gel solution around glass molds. With this method, we obtained reproducible well configurations sensuring that the agarose barrier was consistent in size from experiment to experiment. The first mold (with wells in relatively close proximity; Figure 1A) was particularly suited for adherent cell studies in which cells were observed in a small area at the interface of two wells. This well shape, a 1-mm-thick separation between the well containing the cells and the well containing the growth factor, was designed for rapid diffusion of the chemoattractant molecules and fast induction of cell migration. Furthermore, this thin agarose separation facilitates cell observation. 


\section{Research Reports}

The second mold (with more widely separated wells connected by a channel; Figure 1B) was more suitable for nonadherent cells, because cells in the corridor were easier to observe when dispersed in a larger area than with the previous system or during simultaneous study of different types of adherent cells; the wells were further apart, and their migration toward each other could be more easily evaluated. Moreover, the narrow corridor minimized movement of medium when the dishes were handled for microscopic observation. This is important because movement of medium can make it difficult to detect the start of migration for suspension cells.

\section{Cell Behavior in Agarose Gel}

To characterize cell migration in this assay, we were first interested in how they penetrated the agarose membrane separating the two wells. Cells were observed with a confocal microscope (LSM 510; Zeiss) by taking one picture per hour during 6-24 h. For adherent cells, the migration start was variable in a cell-specific manner and depended on the number of passages for the primary cells. VSMCs began to migrate at the bottom of the separation wall 12-24 h after stimulation with 10\% FBS-DMEM. For VSMCs, fibroblasts, or 1547 cells, movement through the agarose barrier forced the cells to acquire a more compact and spherical shape (Figure 2C) than before, especially for VSMCs and fibroblasts, which are naturally fusiform. After the first cell wave passed through the agarose, adherent cells migrated in several superposed layers. In the following cell waves, cell shape seemed to be less affected by the passage through agarose and speed seemed increased, suggesting that the first cells facilitated the migration of those following. After 4-6 days stimulation, we observed cell polarization toward the chemoattractant source for approximately $40 \%$ of the cells (Figure 2D).

\section{Evaluation of Cell Parameters}

Cell counting. In order to quantify and compare the chemotactic potency of growth factors, it was important to be able to calculate the number
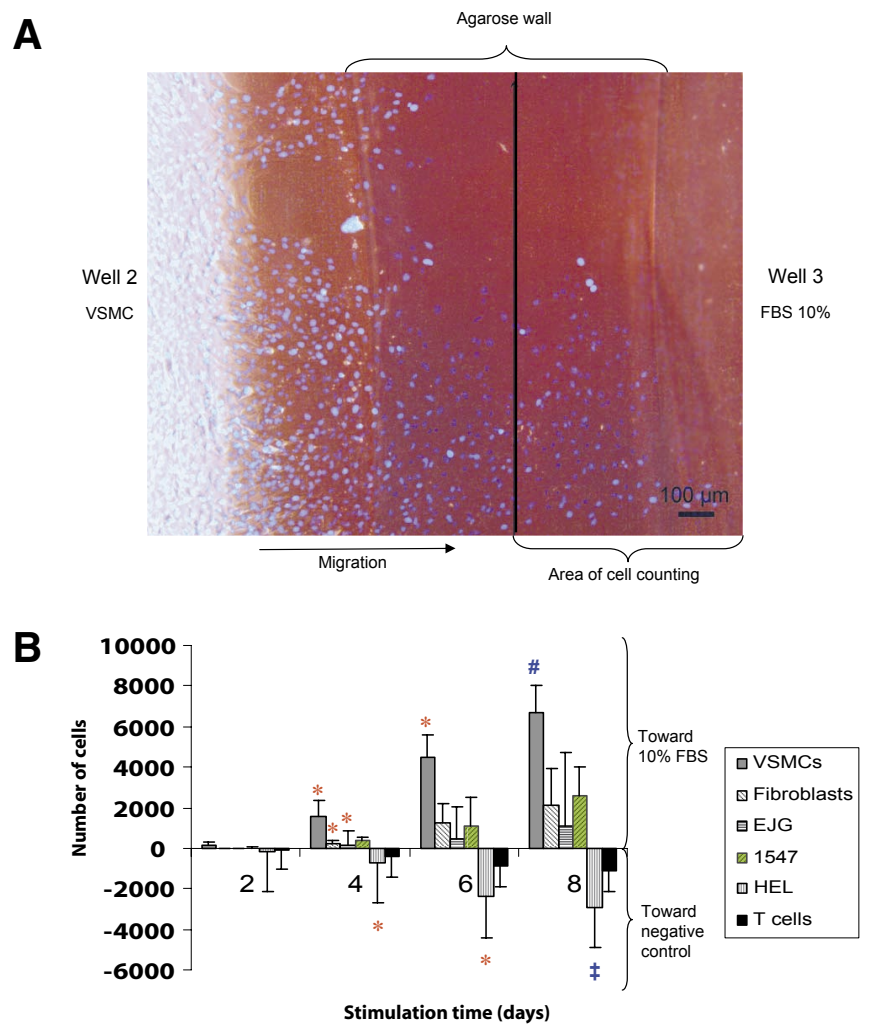

Figure 3. Cell counting. (A) Quantification of adherent cells was done by counting the cells that had reached the half distance separating them from the chemoattractant source (black line). (B) Quantification of migrating cells of different types after 2-8 days of stimulation with 10\% FBS DMEM. For each cell type, the cell number corresponds to the mean \pm SEM of three independent experiments. Negative values represent cells migrating in the opposite direction from the growth factor gradient. (*P $<0.05$, for a given cell type at day $=N$ versus day $=N-2 ; \# P<0.05$, for VSMCs versus fibroblasts, EJG, and HEL; $\ddagger P<0.05$, for HEL versus VSMCs, fibroblasts, EJG, and 1547). FBS, fetal bovine serum; DMEM, Dulbecco's modified Eagle's medium; VSMCs, vascular smooth muscle cells; HEL, human erythroleukemia cells; EJG, bovine capillary endothelial cells.

of migrating cells. Cell nuclei were labeled using the fluorescent DNA dye Hoechst 33258 at a concentration of $50 \mathrm{ng} / \mathrm{mL}$; growth curve experiments confirmed that there was no noticeable effect of the dye on the growth of different cell types at this concentration (data not shown). Using NIS imaging software, migrating adherent cells were counted when they reached a virtual line located in the middle of the agarose membrane separating the wells (Figure 3A). Floating cells were counted when they entered the corridor (Figure 3B). Quantification of cell migration could begin after 2-4 days of stimulation depending on cell type and was normalized by subtracting the number of cells that had migrated to the negative control well (Figure 4). For all tested cells and chemoattractants, induced migration was always significantly higher than random cell movement. CK represented approximately 5\%-10\% CT for adherent cells and 20\%-30\% for floating cells.

As our principal model was VSMCs, gel composition and medium used for the experiments were specifically optimized for them. All cells were studied in DMEM to be able to compare their reactions in the same conditions. We compared the migration of fibroblasts, EJG, 1547 , HEL, and T lymphocytes with that of VSMCs when $10 \%$ FBS was used as the source of chemoattractant (Figure 3B). Observing cell-specific migration, VSMCs migrated toward the $10 \%$ FBS source, while nonadherent cells such as HEL and T lymphocytes also migrated in a time-dependent manner but in the opposite direction (Figure 3B). This appears to be consistent with recent 


\section{Research Reports}
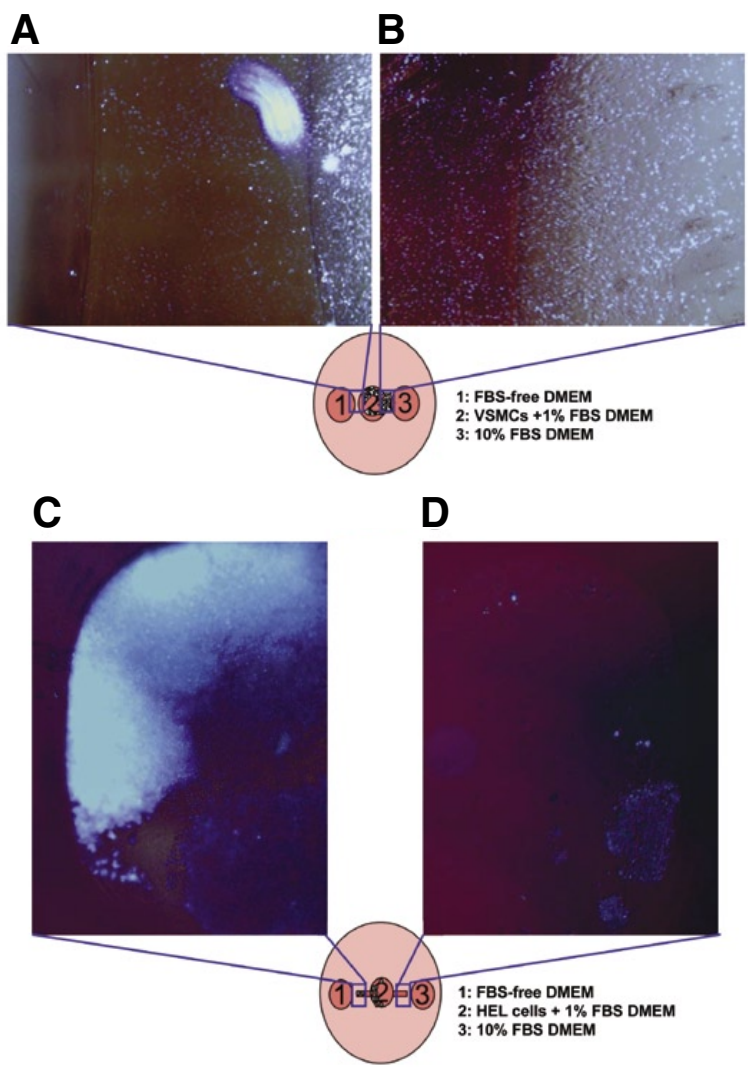

Figure 4. Comparison of chemotaxis (CT) and chemokinesis (CK). Cell migration toward FBS-free DMEM was considered as random migration (CK). Cell migration toward growth factor was considered as specifically directed migration (CT). (A) VSMC migration toward FBS-free DMEM. (B) VSMC migration toward 10\% FBS. (C) HEL migration toward FBS-free DMEM. (D) HEL migration toward 10\% FBS. Cells were visualized using Hoescht nuclear labeling after 6 days of stimulation. FBS, fetal bovine serum; DMEM, Dulbecco's modified Eagle's medium; VSMCs, vascular smooth muscle cells; HEL, human erythroleukemia cell.

data suggesting that after inflammatory activation, $\mathrm{T}$ lymphocytes may be less sensitive to chemoattractant in order to keep their position at the site of primary activation or by the fact that $10 \%$ FBSDMEM contains a repulsive chemokine for $\mathrm{T}$ cells $(26,27)$.

FBS $(10 \%)$ induced a nearly linear increase in number of migrating cells, a trend that could be followed beyond 1 week. We observed that the number of migrating cells increased with incubation time and that VSMC migratory capacity was significantly higher than that of other adherent cells tested (i.e., fibroblast, EJG, or 1547) (Figure 3B).

Cell speed. Using NIS software, it was possible to estimate cell speed. By taking pictures of the same cells during their migration and by comparison of the distance that separated them from the inside of the well where they were seeded (Figure 4A), we could calculate the average estimated speed of a reference sample of cells or of all cells.

In VSMCs, we determined estimated migration speed when cells were exposed to the growth factors bFGF, PDGF-BB, and S1P, which are well known to induce VSMC motility (28-31), and compared their effects to those induced by FBS (Figure 5B). First, with dose-response experiments using PDGF-BB and S1P, respectively, we found $25 \mathrm{ng} / \mathrm{mL}$ and $1 \mu \mathrm{M}$ to be the optimal concentrations for VSMC growth. We hypothesized that the growth factor concentration inducing optimal cell growth would also be the optimal concentration to induce cell migration. After 6-8 days of stimulation, PDGF-
BB-induced migration speed of VSMC was approximately 2 -fold higher than S1P-induced migration. PDGF-BB and S1P together induced a 30\% lower migration speed than PDGF-BB alone. In addition, PDGF-BB did not show a significant difference compared to FBS as a chemoattractant for VSMCs.

\section{Analysis of Migration Induced by Multiple Chemoattractant Gradients}

One interesting feature of our technique is its ability to analyze the behavior toward one chemoattractant versus another or toward a combination of two growth factors used at different concentrations. The simultaneous stimulation of VSMCs with bFGF (25 $\mathrm{ng} / \mathrm{mL}$ ) in the left well and PDGF-BB $(25 \mathrm{ng} / \mathrm{mL})$ in the right one showed that cells responded in an equivalent manner to both stimulations (Figure 6A). We obtained approximately the same quantity (6500-7000) of migrating cells after 10 days of stimulation for both FGF and PDGF (Figure 6B). Migration kinetics were also very similar for bFGF and PDGF-BB: the estimated migration speed increased to $250-300 \mu \mathrm{m} /$ day between day 6 and 8 of stimulation, and after 8-10 days, the speed plateaued.

In these experiments, when cells were submitted to two chemoattractant factors placed in opposing wells (PDGF-BB in well 1 and bFGF in well 3 ), their migration speed was slower than those induced by one factor only (approximately half of FBS-induced migration speed; Figure 6A). When PDGF-BB was used in the singlechemoattractant method, the induced migration speed was comparable to FBS (Figure 5B). This finding suggests that the two-chemoattractant setup may be useful for uncovering differences in chemotactic potency that are not detectable in the single-chemoattractant assay.

This method can also be used to assess chemokine-mediated interactions between two cell populations. By seeding EJG and VSMC, respectively, in wells 1 and 2 of the corridorcontaining well shape, we were able to observe their ability to attract each other (Figure 6C). As these two cell 


\section{Research Reports}

types grew in the agarose wells and secreted growth factors, they were predicted to create complex opposing gradients. After 8 days of co-culture, VSMC and EJG migrated toward each other. VSMC migration speed was 30\% higher than EJG. Similar data were obtained with VSMC and 1547 cells, but with a lesser difference in migration speed (around 10\% higher for VSMC). Using NIS software, it was possible to create a large image by joining different contiguous microscopic fields. The image, shown in Figure 6C, represents an approximate area of $20 \mathrm{~mm}^{2}$. This software feature provides a complete real-time view of the migrating cell population.

\section{DISCUSSION}

We describe a simple-to-perform assay that permits visualization of a migrating cell population and allows analysis of cell morphology, enumeration, and speed estimation. Our agarose assay is applicable to both adherent and suspension cells and can follow migration periods of a few hours to several days. Although the assay is particularly adapted to VSMC, only minor modifications are needed to adapt it to other cell types, and here we show that the method can be used with fibroblasts, human $\mathrm{T}$ lymphocytes, and HEL cells. In addition, we found that EJG and tumor cells such as the osteosarcoma line 1547 or even only their culture supernatant could induce rat VSMC migration after 2-4 days of incubation (data not shown).

We observed that all tested growth factors could induce cell migration, suggesting they were able to diffuse in the agarose; however, modifying the agarose concentration may be useful in situations where diffusion of the chemokine is a problem. The estimated cell speed we observed ranged between 200 and $600 \mu \mathrm{m} /$ day, which is comparable to speed measurements obtained in other studies $(32,33)$. If measured migration speeds for individual cells are desired, a microscope linked to a temperature and atmosphere controlled chamber could be used, enabling continuous data capture.

Even though our assay probably requires more preparation than

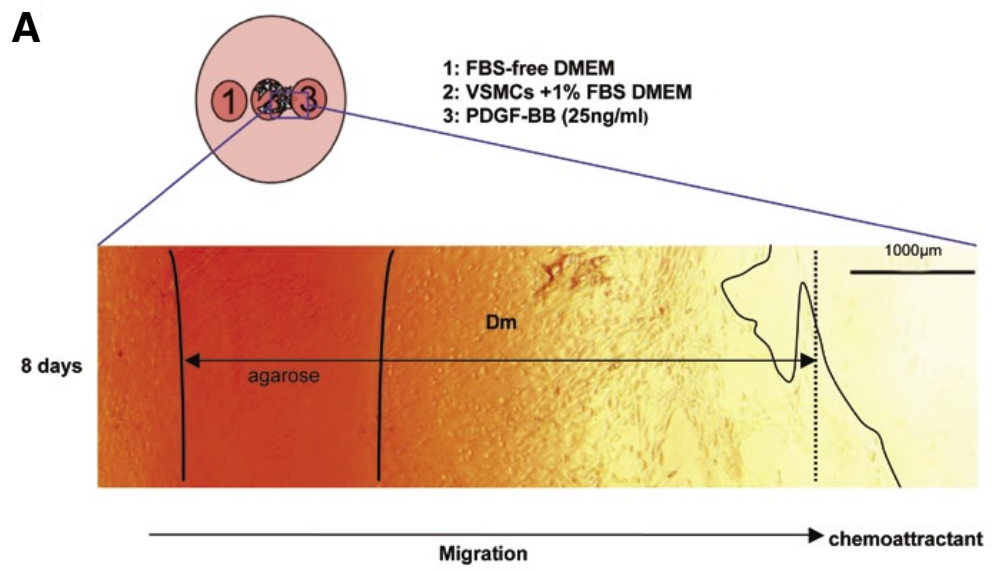

B

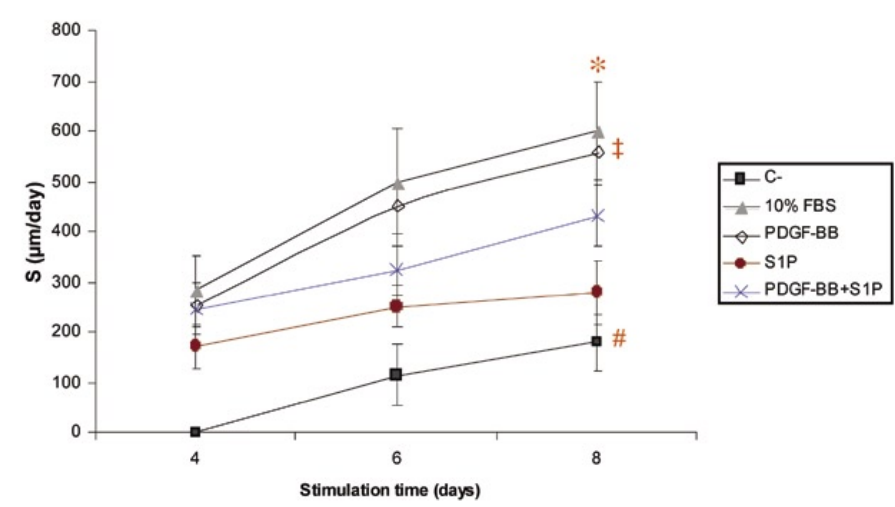

Figure 5. Cell-speed determination. (A) The average speed $S$ was calculated by evaluating the median distance $\left(D_{m}\right)$ of the cell migration front from the internal well border, identified by the dotted line. (B) Speed determination of migrating vascular smooth muscle cells (VSMCs). Average speed was evaluated after 4, 6, and 8 days of stimulation with $10 \%$ fetal bovine serum (FBS), Platelet-derived growth factor (PDGF-BB; $25 \mathrm{ng} / \mathrm{mL}), \mathrm{S} 1 \mathrm{P}(1 \mu \mathrm{M})$, and PDGF-BB $(25 \mathrm{ng} / \mathrm{mL})+\mathrm{S} 1 \mathrm{P}(1 \mu \mathrm{M})$. Each point represents the mean \pm SEM of three independent experiments. ( ${ }^{*} P<0.05$ was found for $10 \%$ FBS compared to C- and S1P $(1 \mu \mathrm{M})$, but not for PDGF-BB $(25 \mathrm{ng} / \mathrm{mL})+\mathrm{S} 1 \mathrm{P}(1 \mu \mathrm{M})$ nor PDGF-BB $(25 \mathrm{ng} / \mathrm{mL})$; \#P<0.05 was found for $\mathrm{C}$ - compared to all growth factors except $\mathrm{S} 1 \mathrm{P}(1 \mu \mathrm{M}) ; \ddagger P<0.05$ was found for PDGF-BB compared to C- and S1P $1(\mu \mathrm{M})$ but not for PDGF-BB $(25 \mathrm{ng} / \mathrm{mL})+\mathrm{S} 1 \mathrm{P}(1 \mu \mathrm{M})$ nor $10 \% \mathrm{FBS})$.

commercial systems, all components necessary for this assay are inexpensive and available in most laboratories. In the electric cell impedence sensing of chemotaxis (ECIS/taxis) method (34), cell enumeration and cell speed can be measured, but sophisticated equipment is needed; specifically, an electrode between the well containing cells and the well containing the chemoattractant is required to detect the resistance increases that indicate when the cells arrive. Similarly, a commercially available cell sedimentation manifold (CSM, Phoenix, AZ, USA) requires specialized equipment.

Although modified Boyden chambers continue to be popular, and the experiment duration is much shorter (30 min to several hours), migrating cells can only be counted after passing through the pores. As a consequence, local chemoattractant-concentration gradients may be modified by the cells obstructing the pore. Moreover, direct cell observation during migration or measurement of cell speed is impossible. In our assay, we could use Hoechst labeling to identify each cell during the entire migration. The use of this DNA stain should also permit easy detection of mycoplasma contamination, which can modify cell motility and is not uncommon in primary cultures.

Another potential limitation of Boyden chambers is that the compet- 


\section{Research Reports}

A

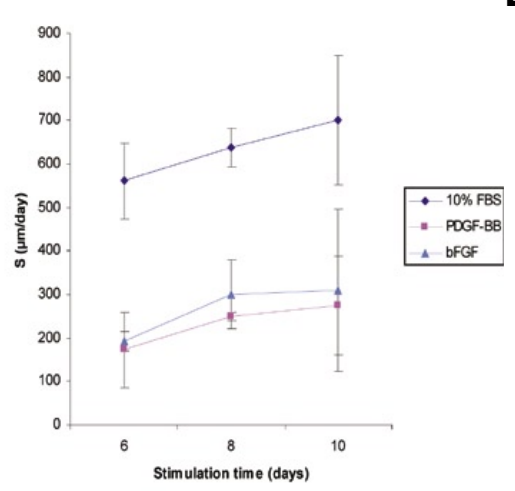

B

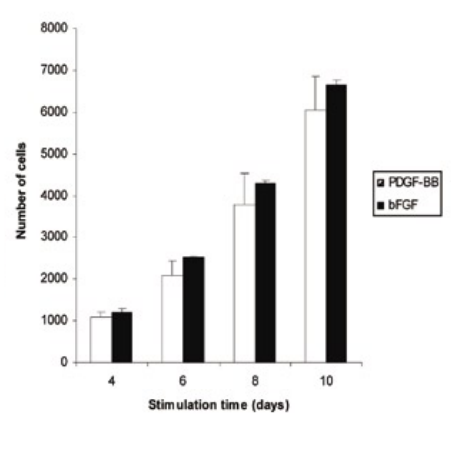

\section{C}

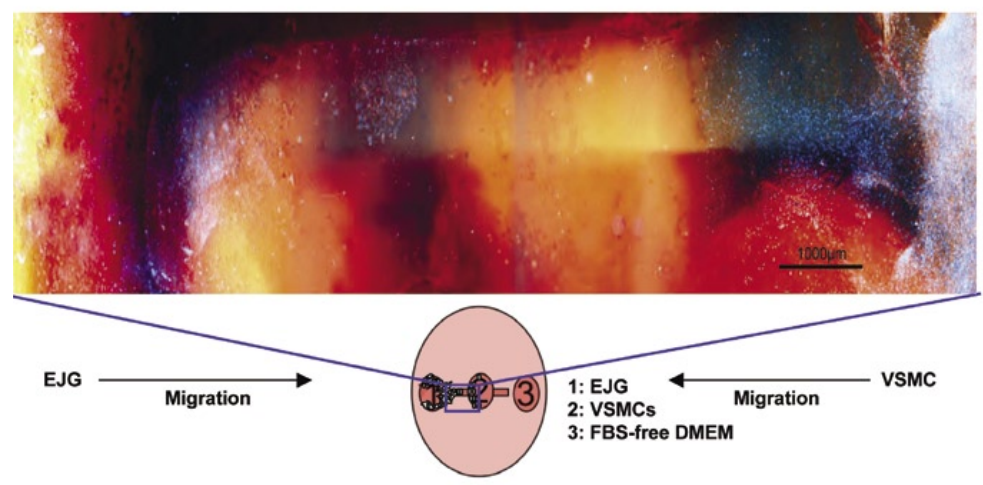

Figure 6. Comparison of simultaneous migration induction. (A) Migration speed induced by simultaneous stimulations with bFGF $(25 \mathrm{ng} / \mathrm{mL})$ in the left well and PDGF-BB $(25 \mathrm{ng} / \mathrm{mL})$ in the right well for 6, 8, and 10 days. bFGF and PDGF-BB induced migrations were compared with the effect of $10 \%$ FBS observed in an independent reference experiment. (B) Quantification of the migrating VSMCs toward each growth factor during the same time lapse. Data represent mean SEM of three separate experiments. (C) Cellular-induced migration between EJG and VSMC. The two cell types migrate toward each other in the corridor separating them. Cells were visualized using Hoescht nuclear labeling after 8 days of coculture. PDGF-BB, Platelet-derived growth factor; VSMCs, vascular smooth muscle cells; EJG, bovine capillary endothelial cells; bFGF, basic fibroblast growth factor.

itive effect between two chemoattractants cannot be studied as the cells can only move in one direction, from the upper to the lower chambers. In our assay, labeled cells could be subjected to different chemoattractant gradients, and we observed that this could have an effect on migration kinetics.

Zigmond and Dunn chambers also enable study of only one cell type versus one chemoattractant at a time. In addition, the chambers are quite fragile and can be easily broken, and the protocol used to seed cells correctly in the chamber is somewhat complicated. Although some technical expertise is required to pour reproducible gels accurately in our assay, the glass molds are not easily broken, and they can be autoclaved and used many times, further reducing the expense.
We describe using our assay to follow early or late effects of growth factors on cells in experiments in which migration is followed for 1-14 days. Since doubling time of the cells was 4 days, we could expect up to two doublings. Thus, some of the cells counted during migration quantification are likely to represent daughter cells instead of cells that had migrated themselves. However, since each single-chemoattractant assay had a negative control well, the number of attracted cells was expressed as the difference of the number of cells migrating toward the chemoattractant versus the number of cells migrating toward the negative control well. If the cell colonization of the neighboring wells was only due to cell growth, we would probably not observe a significant difference between the well containing growth factor and the control well. Thus, cell division may account for a portion of the increase in cell number; however, it is far from being able to describe the entire set of results. We stopped our experiments after 10-12 days because no more significant results were observed beyond that period, but we predict that cells could be maintained in agarose wells longer, especially those with a slow growth rate.

We expect that our method would be compatible with immunohistochemistry experiments that could be performed to assess expression of cytoskeleton proteins, matrix metalloproteinases (35), or growth factor receptors (36) implicated in cell migration. Cells could also be trypsinized at any stage of migration for Western blot analysis.

In summary, our agarose-based migration assay provides valuable data concerning cell migration and chemotactic potency of different factors. We believe it represents an interesting new method to elucidate physiopathological processes, such as vascular wall cell recruitment during tumor angiogenesis and atherogenesis.

\section{ACKNOWLEDGMENTS}

The authors thank Marc Pignot for his interest in this project and for producing the glass templates used in this protocol. We also thank Fondation pour la Recherche Médicale (Comité Limousin) for a generous research grant (to Y.M.).

\section{COMPETING INTERESTS STATEMENT}

The authors declare no competing interests.

\section{REFERENCES}

1. Jain, R.K. 2003. Molecular regulation of vessel maturation. Nat. Med. 9:685-693.

2. Carmeliet, P. 2003. Angiogenesis in health and disease. Nat. Med. 9:653-660.

3. Paik, J.H., S. Chae, M.J. Lee, S. Thangada, and T. Hla. 2001. Sphingosine 1-phosphateinduced endothelial cell migration requires the expression of EDG- 1 and EDG-3 receptors and Rho-dependent activation of alpha vbeta3- 


\section{Research Reports}

and beta1-containing integrins. J. Biol. Chem. 276:11830-11837.

4. Stengel, D., C. O'Neil, I. Brocheriou, S.A. Karabina, H. Durand, N.M. Caplice, J.G. Pickering, and E. Ninio. 2006. PAF-receptor is preferentially expressed in a distinct synthetic phenotype of smooth muscle cells cloned from human internal thoracic artery: functional implications in cell migration. Biochem. Biophys. Res. Commun. 346:693-699.

5. Boyden, D.G. 1970. Cannula for introducing alpha-chymotrypsin into posterior chamber. Am. J. Ophthalmol. 69:1082.

6. Tamama, K., J. Kon, K. Sato, H. Tomura, A. Kuwabara, T. Kimura, T. Kanda, H. Ohta, et al. 2001. Extracellular mechanism through the Edg family of receptors might be responsible for sphingosine-1-phosphate-induced regulation of DNA synthesis and migration of rat aortic smooth-muscle cells. Biochem. J. 353:139-146.

7. Ancelin, M., S. Chollet-Martin, M.A. Herve, C. Legrand, J. El Benna, and M. PerrotApplanat. 2004. Vascular endothelial growth factor VEGF189 induces human neutrophil chemotaxis in extravascular tissue via an autocrine amplification mechanism. Lab. Invest. 84:502-512.

8. Schichor, C., T. Birnbaum, N. Etminan, O. Schnell, S. Grau, S. Miebach, K. Aboody, C. Padovan, et al. 2006. Vascular endothelial growth factor A contributes to glioma-induced migration of human marrow stromal cells (hMSC). Exp. Neurol. 199:301-310.

9. Tamama, K., H. Tomura, K. Sato, E. Malchinkhuu, A. Damirin, T. Kimura, A. Kuwabara, M. Murakami, and F. Okajima. 2005. High-density lipoprotein inhibits migration of vascular smooth muscle cells through its sphingosine 1-phosphate component. Atherosclerosis 178:19-23.

10. Falk, W., R.H. Goodwin, Jr., and E.J. Leonard. 1980. A 48-well micro chemotaxis assembly for rapid and accurate measurement of leukocyte migration. J. Immunol. Methods 33:239-247.

11.Zigmond, S.H. and J.G. Hirsch. 1973. Leukocyte locomotion and chemotaxis. New methods for evaluation, and demonstration of a cell-derived chemotactic factor. J. Exp. Med. 137:387-410.

12. Fabro, G., R.A. Rovasio, S. Civalero, A. Frenkel, S.R. Caplan, M. Eisenbach, and L.C. Giojalas. 2002. Chemotaxis of capacitated rabbit spermatozoa to follicular fluid revealed by a novel directionality-based assay. Biol. Reprod. 67:1565-1571.

13. Zicha, D., G.A. Dunn, and A.F. Brown. 1991. A new direct-viewing chemotaxis chamber. J. Cell Sci. 99:769-775.

14. Campbell, M. and E.R. Trimble. 2005. Modification of PI3K- and MAPK-dependent chemotaxis in aortic vascular smooth muscle cells by protein kinase CbetaII. Circ. Res. 96:197-206.

15. Nelson, R.D., P.G. Quie, and R.L. Simmons. 1975. Chemotaxis under agarose: a new and simple method for measuring chemotaxis and spontaneous migration of human polymorphonuclear leukocytes and monocytes. J. Immunol. 115:1650-1656.
16. Laevsky, G. and D.A. Knecht. 2001. Underagarose folate chemotaxis of Dictyostelium discoideum amoebae in permissive and mechanically inhibited conditions. BioTechniques 31:1140-1142, 1144, 1146-1149.

17. Heit, B., S. Tavener, E. Raharjo, and P. Kubes. 2002. An intracellular signaling hierarchy determines direction of migration in opposing chemotactic gradients. J. Cell Biol. 159:91-102.

18. Heit, B., G. Jones, D. Knight, J.M. Antony, M.J. Gill, C. Brown, C. Power, and P. Kubes. 2006. HIV and other lentiviral infections cause defects in neutrophil chemotaxis, recruitment, and cell structure: immunorestorative effects of granulocyte-macrophage colony-stimulating factor. J. Immunol. 177:6405-6414.

19. Ehrenfeld, P., C. Millan, C.E. Matus, J.E. Figueroa, R.A. Burgos, F. Nualart, K.D. Bhoola, and C.D. Figueroa. 2006. Activation of kinin B1 receptors induces chemotaxis of human neutrophils. J. Leukoc. Biol. 80:117124.

20. Lin, F. and E.C. Butcher. 2006. T cell chemotaxis in a simple microfluidic device. Lab Chip 6:1462-1469.

21. Dee, K.C., T.T. Anderson, and R. Bizios. 1999. Osteoblast population migration characteristics on substrates modified with immobilized adhesive peptides. Biomaterials 20:221-227.

22. Kouvroukoglou, S., K.C. Dee, R. Bizios, L.V. McIntire, and K. Zygourakis. 2000. Endothelial cell migration on surfaces modified with immobilized adhesive peptides. Biomaterials 21:1725-1733.

23. Schichor, C., S. Kerkau, T. Visted, R. Martini, R. Bjerkvig, J.C. Tonn, and R. Goldbrunner. 2005. The brain slice chamber, a novel variation of the Boyden Chamber Assay, allows time-dependent quantification of glioma invasion into mammalian brain in vitro. J. Neurooncol. 73:9-18.

24. Sachinidis, A., M. Flesch, Y. Ko, K. Schror, M. Bohm, R. Dusing, and H. Vetter. 1995. Thromboxane A2 and vascular smooth muscle cell proliferation. Hypertension 26:771-780.

25. Li, A.M., C.A. Vargas, M.A. Brykailo, K.K. Openo, A.H. Corbett, and J.L. FridovichKeil. 2004. Both KH and non-KH domain sequences are required for polyribosome association of Scp160p in yeast. Nucleic Acids Res. 32:4768-4775.

26. Poznansky, M.C., I.T. Olszak, R. Foxall, R.H. Evans, A.D. Luster, and D.T. Scadden. 2000. Active movement of T cells away from a chemokine. Nat. Med. 6:543-548.

27. Ogilvie, P., S. Paoletti, I. Clark-Lewis, and M. Uguccioni. 2003. Eotaxin-3 is a natural antagonist for CCR2 and exerts a repulsive effect on human monocytes. Blood 102:789-794.

28. Fegley, A.J., W.J. Tanski, E. Roztocil, and M.G. Davies. 2003. Sphingosine-1-phosphate stimulates smooth muscle cell migration through galpha(i)- and pi3-kinase-dependent p38(MAPK) activation. J. Surg. Res. 113:3241.

29. Osada, M., Y. Yatomi, T. Ohmori, H. Ikeda, and Y. Ozaki. 2002. Enhancement of sphingosine 1-phosphate-induced migration of vascular endothelial cells and smooth muscle cells by an EDG-5 antagonist. Biochem. Biophys. Res. Commun. 299:483-487.

30. Neeli, I., Z. Liu, N. Dronadula, Z.A. Ma, and G.N. Rao. 2004. An essential role of the Jak2/STAT-3/cytosolic phospholipase A(2) axis in platelet-derived growth factor BB-induced vascular smooth muscle cell motility. J. Biol. Chem. 279:46122-46128

31. Chen, Z., F.Y. Lee, K.N. Bhalla, and J. Wu. 2006. Potent inhibition of platelet-derived growth factor-induced responses in vascular smooth muscle cells by BMS-354825 (dasatinib). Mol. Pharmacol. 69:1527-1533.

32. Scherberich, A., M. Campos-Toimil, P. Ronde, K. Takeda, and A. Beretz. 2000. Migration of human vascular smooth muscle cells involves serum-dependent repeated cytosolic calcium transients. J. Cell Sci. 113:653662.

33. Fukai, N., M. Shichiri, N. Ozawa, M. Matsushita, and Y. Hirata. 2003. Coexpression of calcitonin receptor-like receptor and receptor activity-modifying protein 2 or 3 mediates the antimigratory effect of adrenomedullin. Endocrinology 144:447-453.

34. Hadjout, N., X. Yin, D.A. Knecht, and M.A. Lynes. 2007. Automated real-time measurements of leukocyte chemotaxis. J. Immunol. Methods 320:70-80.

35. Lehti, K., E. Allen, H. Birkedal-Hansen, K. Holmbeck, Y. Miyake, T.H. Chun, and S.J. Weiss. 2005. An MT1-MMP-PDGF receptorbeta axis regulates mural cell investment of the microvasculature. Genes Dev. 19:979-991.

36.ten Freyhaus, H., M. Huntgeburth, K. Wingler, J. Schnitker, A.T. Baumer, M Vantler, M.M. Bekhite, M. Wartenberg, et al. 2006. Novel Nox inhibitor VAS2870 attenuates PDGF-dependent smooth muscle cell chemotaxis, but not proliferation. Cardiovasc. Res. 71:331-341.

Received 18 April 2007; accepted 22 July 2007.

Address correspondence to Franck G. Sturtz, Laboratory of Biochemistry, School of Medicine, University of Limoges, 2, Rue du Dr. Raymond Marcland, F-87025 Limoges, France.e-mail:franck.sturtz@unilim.fr

To purchase reprints of this article, contact: Reprints@BioTechniques.com 